\section{Endocrine disease in parrots}

\section{Yvonne van Zeeland}

Although relatively rare, endocrine disease may occur in parrots. Most often, patients will present with clinical signs that are the result from a hypo- or hyperfunction of one of the endocrine organs.

Pituitary gland disease in birds has primarily been linked to neoplastic processes. Particularly in budgerigars, pituitary tumours are frequently seen. Although these tumours may result in endocrine dysfunction, clinical signs predominantly result from pressure on the surrounding nervous tissue. Imaging techniques (e.g. ultrasonography, computed tomography (CT), magnetic resonance imaging (MRI)) may help to confirm the diagnosis, but owners need to be made aware that, following the diagnosis, no further treatment is available (yet).

Disease of the parathyroid gland is more common in birds. Hyperparathyroidism secondary to the feeding of an all-seed diet, which is deficient in calcium, is commonly seen in parrots. In young, growing birds, this results in rickets, whereby skeletal deformities and fractures may occur, whereas in adult grey parrots, hypocalcaemiaassociated seizures are most noticeable. In both types of patients, correction of the diet, including supplementation with calcium and vitamin D, comprises an important part of the treatment regimen.

A second nutritional disease that can be recognised in birds is goitre. This disease, which is most commonly seen in budgerigars, is characterised by a hypertrophy of the thyroid gland resulting from insufficient dietary iodine intake which (partially) blocks the oesophagus and trachea, thereby resulting in regurgitation and dyspnoea. A tentative diagnosis is usually made based on clinical improvement following supplementation with iodine. Aside from goitre, thyroid neoplasia, hypo- and hyperthyroidism have also been reported in psittacine birds. Of these, hypothyroidism seems most frequently encountered, resulting in clinical signs such as obesity and alopecia. In many of the reported cases, diagnosis was made based on successful response to thyroxine supplementation. Confirmation of the disease will, however, necessitate the use of a thyroid-stimulating hormone (TSH) stimulation test, following which supplementation of thyroxine can commence. In practice, hypothyroidism can often be ruled out easily by examining the bird: if new, developing feathers are seen, hypothyroidism is highly unlikely, as lack of growth of new feathers is a characteristic of endocrine alopecia.

Adrenal disease has been reported very rarely in birds, with only one antemortem case of Cushing's syndrome described in the literature. Following diagnosis, this patient, a 12-year-old Senegal parrot, which presented with severe obesity, polyphagia, polyuria/polydipsia and mild alopecia, was successfully treated with trilostane for over a year.
Diabetes mellitus will usually result from a dysfunction of the endocrine pancreas, although in birds it is unsure whether the disease originates from a lack of insulin or an excess of glucagon. This disease, which is most commonly encountered in smaller psittacines, results in severe hyperglycaemia and glucosuria. Treatment with insulin has been described, but is usually challenging.

In conclusion, endocrine diseases do occur in psittacine birds, but may be underdiagnosed due to the lack of, or unfamiliarity with, appropriate testing. The latter is, however, extremely important for obtaining a definite diagnosis and initiating a proper therapeutic plan.

\section{KEY LEARNING OBJECTIVES}

- Endocrine disease does occur in psittacine birds, but may be underdiagnosed due to lack of or unfamiliarity with, appropriate testing methods.

- If endocrine disease is suspected based on the clinical signs, every effort should be made to confirm the disease prior to initiating a treatment plan. In this light, function tests are considered particularly useful

- Diagnosis and treatment of endocrine disease in birds roughly follow similar guidelines as those of other species

\section{MULTIPLE CHOICE QUESTIONS}

1. Pituitary tumours are most commonly diagnosed in which of the following?
a. Amazon parrots
b. Budgerigars
c. Cockatiels
d. Macaws

2. Which of the following endocrine diseases is most commonly encountered in psittacine birds?
a. Cushing's syndrome
b. Diabetes mellitus
c. Hyperparathyroidism
d. Hypothyroidism

3. Which of the following clinical signs is most commonly seen in birds with goitre?
a. Alopecia
b. Dyspnoea
c. Obesity
d. Regurgitation

4. In birds, diagnosis of diabetes mellitus is made following the finding of a fasting blood glucose level in excess of which value?
a. $10 \mathrm{mmol} / \mathrm{l}$
b. $18 \mathrm{mmol} / \mathrm{l}$
c. $25 \mathrm{mmol} / \mathrm{l}$
d. $44 \mathrm{mmol} / \mathrm{l}$

5. What is diagnosis of hypothyroidism in birds based on?

a. The typical clinical signs (obesity and alopecia with lack of feather growth)

b. Finding of low plasma T4 levels

c. Lack of response to stimulation with TSH

d. Resolution of clinical signs following thyroxine supplementation 